\title{
Identification d'un gène de la sclérose tubéreuse de Bourneville
}

La sclérose tubéreuse de Bourneville est une affection autosomique dominante, classée parmi les phacomatoses, caractérisée par la formation de tumeurs appelées hamartomes $(\mathrm{m} / \mathrm{s}$ $n^{\circ} 9$, vol. 7, p. 978). Cellesci apparaissent dans de nombreux tissus, expliquant la variété des symptômes et des complications. Deux temps pourraient être nécessaires à l'apparition de la maladie clinique: une perte d'hétérozygotie pour un des allèles, observée effectivement dans certains hamartomes, suivie d'une mutation somatique. Il serait ainsi nécessaire de parvenir à l'inactivation des deux allèles pour que les symptômes se manifestent. Des travaux antérieurs de génétique avaient proposé l'existence de deux loci génétiques, sur le chromosome 9 en 9q3a.5 (TSC1), et sur le chromosome 16 en $16 \mathrm{p} 13$ (TSC2). Le gène situé sur le chromosome 16 vient d'être identifié par le consortium européen créé à cet effet (Cardiff et Oxford, GrandeBretagne, Rotterdam et Leyde, PaysBas, Lisbonne, Portugal) [1]. On a précisé la position de cinq délétions dans une région de $120 \mathrm{~kb}$. Elle a été clonée dans des cosmides et quatre gènes ont été isolés. L'un d'eux, dénommé TSC2, est interrompu par chacune des délétions. Son transcrit, qui mesure $5,5 \mathrm{~kb}$, a une large expression dans de nombreux tissus. La séquence de la protéine codée, la tubérine, comportant 1784 acides aminés, est dépourvue de peptide signal et fait prévoir la présence de $m / s \quad n^{\circ} 5$ vol. 10, mai 94 quatre segments transmembranaires et de glissières à leucine. Elle présente une région de ressemblance avec la protéine stimulant l'activité GTPasique des protéines de la famille Ras, appelée GAP3 (GTPase activating protein 3). A ce titre, la lésion moléculaire de la sclérose tubéreuse de Bourneville évoque celle d'une autre phacomatose, la neurofibromatose de type 1 , dont le gène NF1 code pour une autre protéine GAP $\left(m / s n^{\circ} 4, v o l .8, p .471\right)$. Dans ce cas, divers auteurs ont proposé que la diminution de l'activité GTPasique de la protéine NF1, ou l'absence de cette protéine, perturberait la désactivation de Ras-GTP liée à sa transformation en Ras-GDP. Or, Ras étant une plaque tournante dans la transmission de nombreux signaux de prolifération, la perte du contrôle négatif de son activité peut aisément expliquer la survenue des tumeurs. Des travaux complémentaires devraient déterminer si un tel mécanisme peut être évoqué pour GAP3, et, dans ce cas, quelles sont les bases des différences des tableaux cliniques de la neurofibromatose de type 1 et de la sclérose tubéreuse de Bourneville : différences de répartition tissulaire, spécificité pour des protéines Ras distinctes...? Quoi qu'il en soit, les gènes $N F 1$ et $T S C 2$, codant pour les deux protéines GAP, se comportent comme des gènes suppresseurs de tumeur; il est intéressant de noter à cet égard qu'une perte d'hétérozy- gotie pour des allèles situés sur le bras court du chromosome 16 a été observée récemment dans des hamartomes ou angiomyolipomes rénaux accompagnant la sclérose tubéreuse. Enfin, on peut rappeler qu'un des loci de la polykystose rénale autosomique dominante, PKT) 1, ( $m / s n^{\circ} 12$, vol. 9, p. 1426) est localisé dans la même région que TSC2, et que des kystes rénaux multiples peuvent être observés dans la sclérose tubéreuse (mais des kystes rénaux ont également été détectés dans une famille liée à TSC1).

En plus des cinq délétions principales, l'examen de l'ADN de 260 malades supplémentaires a montré dans cinq cas des délétions plus petites. Il est probable que des méthodes plus fines détecteront d'autres types d'anomalies moléculaires dans le gène. Un travail analogue devra être accompli pour l'autre locus de la sclérose tubéreuse situé sur le chromosome 9.

J.C.D. J.P.G.

1. European consortium on tuberous sclerosis on chromosome 16. Cell 1993; 75 : 1305-15.
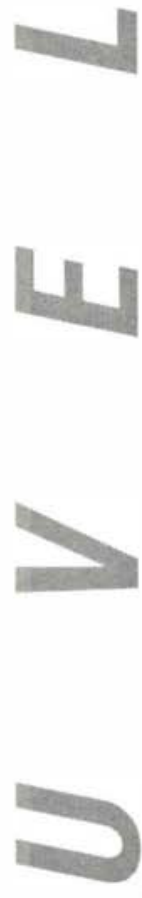